P63 (continued)

at home and completed questionnaires about their feeding practices. The video was coded for parental feeding practices.

Outcome Measures and Analysis: Questionnaire responses of "never," "rarely" were considered correct if the behavior was not observed in the video; responses of "most of the time," "always" were correct if the behavior was observed at least once during dinner. "Sometimes" responses were excluded from analysis. Behaviors that corresponded $>70 \%$ of the time were considered to be reported accurately.

Results: Parents accurately reported the following behaviors: allowing child to eat as much as he wants, helping child to eat, using neutral prompts to eat, having television/screen on during meal, offering non-food rewards for eating, and hurrying child to eat faster. Parents' responses were not accurate for: using reasoning to convince child to eat, allowing child to choose foods, asking child to clean his plate, and offering food rewards for eating.

Conclusions and Implications: Possible explanations why some behaviors are more accurately reported will be discussed. Behaviors which parents can report accurately can be addressed directly through interventions because parents are already aware of using these mealtime behaviors. For less accurately reported behaviors, additional awareness may need to be created through education before parents will be able to recognize and change their behaviors. Funding: Nestle Nutrition

\section{P64 An Exploratory Look at the Role of Childcare Providers as a Support and Resource for Breastfeeding Mothers}

Alexandra Lundquist, BS, lundqui2@illinois.edu, University of Illinois at Urbana-Champaign, 1105 West Nevada, Urbana, IL 61801; Brent McBride, PhD, University of Illinois at Urbana-Champaign;

Sharon M. Donovan, PhD, RD; Chigozirim Asonye;

Alexandra Kieffer; Aubrey Toosley

Objective: Research has examined workplace, healthcare, and family factors in support for the breastfeeding mother, whereas few studies have considered the available support in childcare settings. Breastfeeding continuation in the childcare setting involves the co-commitment and active cooperation between mother and provider. We hypothesized that the transition to non-parental care represents a vulnerable change for first-time mothers where increased support may be needed to facilitate the continuation of breastfeeding, and that mothers overlook childcare as a potential source of influence and support.

Study Design, Setting, Participants, Intervention: Utilizing Bronfenbrenner's Ecological Model as a theoretical framework for the development and evolution of the breastfeeding experience, a semi-structured interview was developed to guide data collection. First-time mothers $(n=25)$ were recruited for interviews.
Outcome Measures and Analysis: Concepts and themes that comprise mothers' experiences with, and perceptions of, navigating the transition to childcare while breastfeeding were identified using Thematic Analysis.

Results: Initial findings suggest that upon the transition to non-parental care, there is a shift in the breastfeeding relationship from one with the infant to one with the breast pump that mothers find less rewarding. Further, mothers do not critically evaluate childcare providers' support for breastfeeding, having only superficial conversations with providers regarding breastfeeding.

Conclusions and Implications: These results demonstrate the need for education that empowers mothers to find support in their childcare provider and provides resources for improving the breastfeeding knowledge of providers. Findings also underscore the need to recognize childcare as a viable source of support for the breastfeeding mother.

Funding: Illinois Transdisciplinary Obesity Prevention Program, National Dairy Council

\section{P65 Are Children Who Attend Family Child- Care Homes Meeting Dietary Recommendations at Home?}

Noereem Mena, MS, LDN, RD, mena23@uri.edu, University of Rhode Island, 125 Fogarty Hall, 41 Lower College Roadd, Kingston, RI 02881; Maggie Tsai, BS, University of Rhode Island; Patricia Risica, DrPH, Brown University; Kim M. Gans, $P h D$, University of Connecticut; Ingrid Lofgren, $P h D, M P H, R D$, University of Rhode Island; Kathleen Gorman, PhD; Alison Tovar, PhD, MPH

Objective: To describe food consumption of children (2-5 years) who attend family child-care homes $(\mathrm{FCCH})$ at home, relative to dietary guidelines.

Study Design, Setting, Participants: Parents of children in FCCHs currently enrolled in a cluster randomized intervention were recruited to participate in an ongoing sub-study to capture foods and beverages their children consume at home.

Outcome Measures and Analysis: Parents $(n=18)$ reported the foods/beverages their children consumed from pickup time to bedtime via two 24-hour recalls. One-sample $\mathrm{t}$ tests and Wilcoxon Signed-rank test, with Bonferroni corrections, were run to test for differences between food groups consumed outside of the FCCH compared to dietary guidelines, assuming that one-third to one-half of total daily nutrient intake should be consumed away from the FCCH. Outcome variables included total energy, refined and whole grains, fruit, and vegetable intake.

Results: Foods consumed outside of child-care are exceeding recommendations for total energy intake, added sugar and refined grains $(\mathrm{p}<0.0001)$. Intake of whole grains and fruit also exceeded recommendations $(\mathrm{p}<0.0001)$, however, vegetable intake fell below both the one-third and one-half proportion recommendations for consumption outside of child-care, mean intake of 0.27 cups vs. recommended intake of 0.3-0.5 cups, $\mathrm{p}<0.0001$.

Conclusion and Implications: Excess energy intake is occurring outside of the FCCH. Yet, children are still not

Continued on page $S 49$ 
P65 (continued)

meeting recommendations for vegetable intake. Future child-care based interventions should consider targeting dietary intake outside the child-care setting as it may have a significant role in obesity risk.

Funding: National Heart Lung and Blood Institute

\section{P66 Challenges and Barriers to Providing Healthy Lifestyle Opportunities in Child Care Centers}

Cynthia A. Warren,PhD, cwarren2@twu.edu, Texas Woman's University, 1200 Frame Street, Denton, TX, 76209; Katherine K. Rose, PhD, Texas Woman's University

Objective: Child care provides an optimal environment to influence young children's relationship with nutritious food and physical activity. More than two-thirds of children between 3 and 5 years of age attend some form of child care each week. The purpose of this study was to obtain in-depth information through focus group discussions to understand child care centers' directors, teachers and staff perceived challenges and barriers to providing healthy lifestyle opportunities to preschool children in their care.

Study Design, Setting, Participants, Intervention: A list of licensed center-based programs in Denton County (TX) was generated from the Texas Department of Family Services website $(n=234)$ and each center mailed a recruitment flyer. Resulting audio-taped sessions were transcribed verbatim.

Outcome Measures and Analysis: Five focus groups, lasting approximately 50 minutes each, were conducted with 46 Denton County (TX) child care providers (teachers, directors, cooks, and owners). The resulting transcripts were read, data unitized, coded and compared, thematically categorized and interpreted using a grounded approach.

Conclusions and Implications: Time, convenience, cost and lack of nutrition knowledge were major underlying themes emerging from these discussions. Findings indicate the lack of affordable, evidence-based nutrition education materials available for child care providers, parents and preschoolers. Additional findings include educating parents and providers on how to prepare budget-friendly nutritious foods for children, as well as easy-to-use methods for getting children to try new foods, was another finding. Study findings will inform future intervention research that promotes the healthy development of young children.

Funding: Texas Woman's University

\section{P67 Child Centered Nutrition Phrases: Messaging Increases Young Children's Consumption of Healthful Foods}

Jane Lanigan, PhD, jlanigan@wsu.edu, Washington State University, 14204 NE Salmon Creek Avenue, Vancouver, WA 98686; Rachel Bailey, PhD, Washington State
University; Samantha Ramsay, $P h D, L D N, R D$, University of Idaho; Valerie Jarvensivu, MS, Washington State University

Objective: Communication strategies that promote young children's healthful eating have received limited empirical study, particularly in early childhood education (ECE) settings. This study examines the effect of developmentally appropriate nutrition phrases on young children's food behavior.

Study Design, Setting, Participants: Eighty-seven children aged 3-6 years attending two ECE programs participated in a within-subject experiment. For the sixweek taste activity intervention, each child was randomly assigned a repeated exposure (RE) and a child-centered nutrition phrase (CCNP) food (quinoa, lentils, tomato, or bell pepper).

Outcome Measures and Analysis: A consumption trial measured individual intake pre- and post-treatment and at one month post-treatment. Grams consumed data were log-transformed then submitted to a 2-level random effects model with time of assessment, phrase condition, their interaction, pre-preference for the foods, and pre-exposure to the foods at level 1; and site, child age, income, parent education level, and parent nutrition knowledge at level 2. Results: Consumption of the CCNP food across the sample increased more than foods using RE alone. Children exposed to the CCNPs consumed $8.95 \mathrm{~g}$ more of their target food post-treatment and $17.9 \mathrm{~g}$ more at the one month post-assessment. A site effect was detected. Children from one ECE program accounted for the increased consumption of the CCNP food within the sample. Children attending the second site consumed less of the CCNP food than the RE food.

Conclusion and Implications: Strategic use of developmentally appropriate nutrition messaging in ECE programs may be an effective strategy for increasing food consumption, but further research is needed to better understand contextual factors.

Funding: University of Technology, Jamaica Research Development Fund

\section{P68 Culturally Relevant Nutrition Education for Migrant Head Start Parents 2016}

Imelda Galdamez, MSW, imeldagaldamez@gmail.com, Michigan State University Extension, 446 West Circle Drive, Room 257, East Lansing, MI 48824;

Courtney Cuthbertson, PhD, Michigan State University Extension; Andrea Aguilar, MS

Objective: Michigan State University Extension implemented a process to design and deliver a culturally and linguistically relevant program to seasonal and migrant workers who were parents of young children during summer 2016.

Description: Michigan State University Extension partnered with a migrant worker service agency to provide culturally-relevant nutrition and physical activity education to seasonal and migrant worker parents with 